\title{
Actions to increase the reliability of the chain transmissions
}

\author{
Ștefan Ghimiși ${ }^{1 *}$ and Dana Nicula ${ }^{2}$ \\ ${ }^{1}$ Constantin Brancusi University of Targu Jiu, Calea Eroilor nr.2, Gorj, România \\ ${ }^{2}$ The Bucharest University of Economic Studies, România
}

\begin{abstract}
Correct analysis of a malfunction is a very important step in the remediation process of a product. It is necessary to have a clear vision, especially to determine the cause of the malfunction, although sometimes it can be masked by other defects. Quality Management offers many classic and modern tools to help find solutions that ensure quality improvement. This paper presents a modern method of quality management, called the tree diagram, applied to the issue of increasing the reliability of the chain transmissions. The advantage of using this chart is that it offers the possibility of logically and chronologically examining the objectives and actions that solve a quality or non-quality problem. In the proposed case study, the overall objective is to increase the reliability of the chain transmissions. Specific objectives are set and for each specific objective, we identify potential solutions that can be adopted. For the proposed general objective, the following three specific objectives have been identified: Reduction of chain wheel wear and chain joints, Ensuring proper fitting, Adoption of optimal chain tensioning solutions. On the basis of the presented measures, the tree diagram was made on three levels. The tree chart made outlines the relationship between the overall goal pursued and the actions taken to achieve them. This tool can be used in planning and solving quality assurance problems in the case of the chain transmissions.
\end{abstract}

\section{Introduction}

Correct analysis of a malfunction is a very important step in the process of repairing a piece or product. It is necessary to have a clear vision, especially to determine the cause of the malfunction, although sometimes it can be masked by other defects. An examination of all defects on a spare/subassembly/product may lead to the correct identification of all the causes and actions to be taken to eliminate the causes of the malfunctions. Quality Management offers many classic and modern tools to help find solutions that ensure quality improvement.

In many situations, numerical data is rarer, and then quality issues cannot be solved by analytics. Thus, non-numerical methods (the 7 new tools of quality management) are used, in which case the problem, the causes determining a non-quality problem, solutions for

\footnotetext{
*Corresponding author: ssghimisi@gmail.com
} 
solving the analysed problem, etc. are identified. Non-numeric data can be transformed into different types of graphs that provide the possibility of a comparative analysis, highlighting trends or establishing relationships between different elements of the problem studied.

This paper presents a modern method of quality management, called the tree diagram, applied to the issue of increasing the reliability of the chain transmissions. The advantage of using this chart is that it offers the possibility of examining logically and chronologically the objectives and actions that solve a quality or non-quality problem. Non-qualitative issues and case studies that apply the quality management tools (for example, fish bone diagram) are outlined in several papers $[1,2,3,4]$. The tree chart was used to analyse defects and establish measures to eliminate nonconformities in many areas of activity (industrial and service activities). The paper [5] illustrates how event tree diagrams used in safety engineering can be applied to test the design of a healthcare service. A water quality study was conducted on the basis of the decision tree and is presented in the paper [6]. Islam presents [7] a methodology based on the tree diagram that determines the dimensional requirements of a product according to the needs of the clients. In the paper [8] there are presented quality tools used by companies wishing to explicitly manage R \& D. Another interesting application is given in the paper [9] which shows the combined use of the fish bone diagram and the tree diagram by managers who develop program proposals and who want to improve the quality of program proposals.

With regard to the chain transmissions, many studies have been published on their defects as well as issues concerning the quality of the chain transmissions. In the paper [10] we present a study on the reliability of the maximum transmission capacity of the chain transmission and propose a mathematical model to optimize the chain transmission system design. The importance of the chain transmission control is a theme in many specialized works. Thus, in the paper [11] there are experimental investigations regarding the chain transmission defects from a Bucket wheel excavator. In the paper [12], a simplified model of a motorcycle rear suspension with the chain transmission is proposed and its stability in equilibrium configurations is studied through eigenvalue analysis. In his paper, Jula [13, 14] analyses some aspects concerning the chain transmissions.

\section{Methods}

In this paper we propose a study on the application of the tree diagram for a mechanical problem. The problem is "increasing the reliability of the chain transmissions", which is also the general objective, as required by the working document for the tree diagram. Specific objectives are set and for each specific objective, identifying potential solutions that can be adopted. The method presented in this paper highlights the relationships between the objectives pursued and the solutions/actions proposed for achieving the objectives.

It is desirable to create a tree diagram that provides a picture of solutions to the problem of increasing the reliability of the chain transmissions. In this case, the tree diagram is a functional analysis that answers the question HOW? act to increase the reliability of the chain transmissions. Aspects of the problem are detailed from general to particular, starting from a general goal set and then developing specific (or primary) measures and secondary measures.

For drawing the tree diagram, the classical method is used which provides for the following steps, according to the SR ISO 9004-4:1996 standard and the instructions in the paper [15], will be followed:

1. Define the topic(s) for which solutions are being solved;

2. Organizing a Brainstorming session with specialists in the field of the problem studied; 
3. The solutions proposed by the specialists are registered as to: How can chain reliability improve?

4. The proposed solutions are grouped into several objectives;

5. Develop the diagram with new possible solutions for achieving the objectives.

\section{Results. The tree diagram to increase the reliability of the chain transmissions}

Identifying the forms of damage to the chain transmissions and determining the causes that cause them allow designers to avoid or limit their effects and thus comply with the conditions of safe operation within a set period of time. It will highlight both forms of damage that can be avoided by resistance calculations as well as by technological and functional measures. It is known from industrial practice that a series of defects may occur during the operation of chain-drive systems. Identifying and eliminating the causes of defects is important because it increases the reliability of the chain transmissions. Based on the study of this topic in various specialized papers, we identified the main factors influencing the reliability of chain transmissions, namely: Mounting position, Chain extension, Chaining of the chain transmissions.

For the purpose of drawing up the tree diagram, we proposed as a general objective: Increasing the reliability of the chain transmissions. For the general objective, we proposed the following three specific objectives as well as the appropriate solutions/measures:

Specific Objective 1. Adopt optimal chain extension solutions

- Tensioning system with stretching wheels;

- Tensioning system with external tire;

- Tension and spring wheel tension system, respectively counterweight;

- Tensioning system with tensioning wheel placed inside;

- Path patch system;

- Type rotation oval;

- High-action skateboard and hydraulic drive;

- Stretcher with stretchers or stretchers.

Specific objective 2. Reducing the wear of chain wheels and chain joints [16]

1. Choose an appropriate lubrication system, taking into account chain speed, transmission location and operating conditions;

- Lubrication system by immersing the chain in the oil bath $(\mathrm{v}<7 \mathrm{~m} / \mathrm{s})$;

- Valve disk-type lubrication system ( $\mathrm{v}>10 \mathrm{~m} / \mathrm{s})$;

- Oil circulation lubrication system (at high loads and very high speeds);

- Dust lubrication system (average speeds);

- Lubrication system by introducing solid grease into the chain joints (medium speeds);

- System with periodic lubrication by liquefaction $(\mathrm{v}<1 \mathrm{~m} / \mathrm{s})$;

2. Choosing suitable materials for both chain and chain wheels

- Chains are made of: carbon-containing steels, cementitious steels, iron-reinforced steel;

- Sheets are made of quality carbon steel or alloy steels for improvement;

- Bolts, bushings and rollers are made of quality carbon steel or alloyed cement casting.

Specific Objective 3. Ensure a proper fit

- To adopt the optimal horizontal position of the centre line;

- Does the vertical position of the transmission;

- It can adopt the inclined position of the centre line to the horizontal with an angle of $30 \ldots 60$. 
A tree diagram for the proposed problem has been developed - Increasing the reliability of the chain transmissions. The proposed problem, which is also the general objective, is the first level of the chart. For the second level of the chart we proposed the three specific objectives identified: Reducing the chain wheel wear and chain joints, Ensuring a proper fitting and Adopting optimal chain tensioning solutions. The appropriate measures proposed for each of the three objectives are Level 3 of the Diagram. The tree diagram is shown in Figure 1.

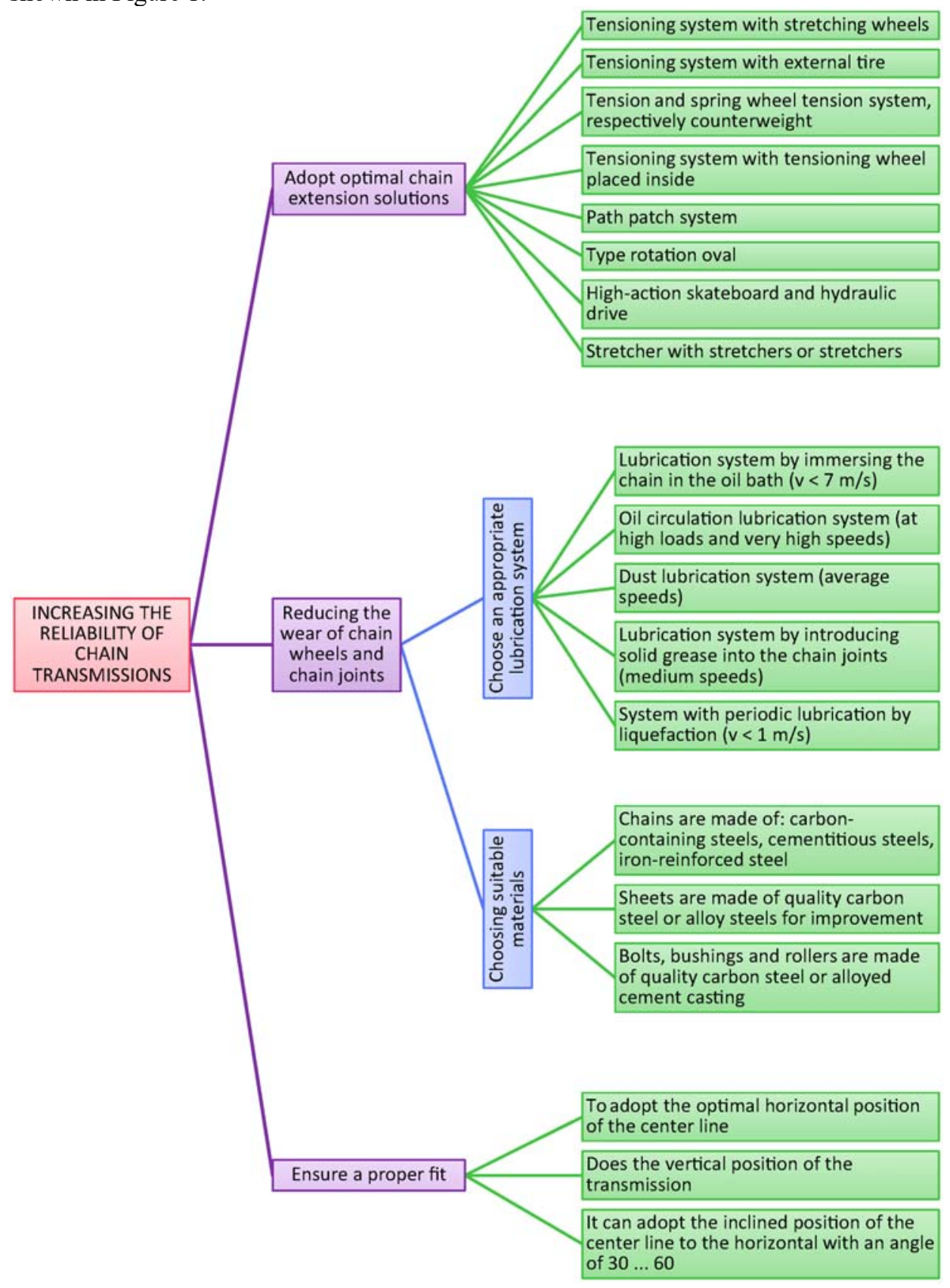

Fig. 1. The tree diagram. 


\section{Discussion}

In the tree diagram of Figure 1, a series of steps have been taken to increase the reliability of the chain transmissions. The measures have been grouped on three specific objectives based on the analysis of the causes that cause the transmissions to come out of operation. When the chain transmission goes out of service, there is no proper gearing of the chain tooth, the chain length increases, the noise increases, the operation is no longer uniform. In particular, the damage occurs as a result of the destruction of the functional surfaces: the chain components and the chain wheel teeth. The proposed measures are constructive and exploitation measures and have been grouped on the basis of three specific objectives proposed to increase reliability. When determining the three objectives, the main causes that caused the damage were: the lubrication system, the chain and chain wheel materials, the tensioning device, the mounting position of the transmission.

Since the major problems in the operation of the chain transmissions are caused by the wear of the chain and wheel joints, it has been established that a specific objective may be "Reducing the wear of chain wheels and chain joints". Two directions of action have been set up for this objective: the choice of a suitable lubrication system; choosing suitable materials for both chain and chain wheels. In order to avoid pronounced wear, there must be a resistant lubricant film between the surfaces in contact, the effective pressure in the film being limited to a permissible value. When choosing lubrication systems, the demands and speeds of the transmissions are taken into account. The reduction of the chain wheel teeth wear can be achieved by improving lubrication conditions. The chain tooth chain and chain teeth wear can be reduced by the proper choice of material and treatment for them, in correlation with the demands on the transmissions. In this case, the measures concern the appropriate choice of wheel, bolt, bolt, bush and roller materials, which must have been called harnesses after the applied thermal treatments.

During the operation of the transmission, the joints wear out, the chain elongates and there must be a device that adjusts the chain tension to compensate for the chain elongation. The designer must adopt a stretch device depending on the functional condition of the transmission. This is a very important aspect and so the chart has been defined as a specific objective - "Adopting optimal chain extension solutions".

Based on the findings from the operation of the chain transmissions, the vertical positioning of these transmissions has to be avoided. In the vertical position there is a tendency for the chain links to come out of the wheel tooth engagement, and in this case the adjustment of the chain is much more demanding. Thus, the optimal horizontal position of the centreline is recommended, or in some special situations an inclined position of the centre line to the horizontal. From this point of view, a third specific objective was included in the diagram - "Ensure a proper fit".

\section{Conclusions}

The tree diagram outlines the relationships between the objectives pursued and the actions taken to achieve them. The use of this diagram allows going from a given theme and then investigating possible solutions that are then evaluated using efficiency, feasibility criteria, etc. This tool can be used in planning and in solving quality assurance issues in many areas of activity. The case study presented demonstrates that this tool is intended to be used to solve the increase in reliability of the chain transmission. The specific objectives and actions identified for each objective are suitable for a graphical representation of the tree diagram type. The grouping of measures on the three main objectives offers the possibility to focus specialists by focusing on the specific problems of each stage in the manufacture and assembly of the chain transmissions. 


\section{References}

1. L.M. Cirtina, D. Cirtina, L. Luca, Applied Mechanics and Materials, Trans. Tech. Publications, Switzerland, 657, 891-895, (2014)

2. L. Luca, L.M. Cirtina, A. Stancioiu, Applied Mechanics and Materials, Trans. Tech. Publications (Switzerland), 657, 256-260, (2014)

3. L. Luca, C. P. Filip, $7^{\text {th }}$ International Conference the Knowledge- Based Organization, Conference Proceedings 1: Management and Military Sciences, Book Series: Knowledge Based Organization International Conference, 691-696, (2011)

4. L. Luca, IManEE 2016, Greece, IOP Conf. Series: Materials Science and Engineering, 161, (2016)

5. G. Despotou, R.W. Jones, T.N. Arvanitis, Unifyng the Applications and Foundations of Biomedical and Health Informatics. Book Series: Studies in Health Technology and Informatics, 226, 119-122, (2016)

6. S.M. Saghebian, M.T. Sattari, R. Mirabbasi, M. Pal, Arab. J. Geosci., 7, 4767-4777, (2014)

7. M.N. Islam, Int. J. Adv. Manuf. Tech., 23, 489-494, (2004)

8. M.M. Menke, Res. Technol. Manage., 37, 25-32, (1994)

9. S.S. Li, L.C. Lee, Res. Evaluat., 20, 275-282, (2011)

10. G.T. Long, $4^{\text {th }}$ International Conference on Materials Engineering for Advanced Technologies (ICMEAT 2015), London, England, 103-106, (2015)

11. S.M. Bosnjak, D.B. Momcilovic, Z.D. Petkovic, M.P. Pantelic, N.B. Gnjatovic, Eng. Fail. Anal., 35, 462-469, (2013)

12. S. Sorrentino, L. Leonelli, Vehicle Syst. Dyn., 55, 1707-1730, (2017)

13. A. Jula, E. Chisu, M. Lates, Mechanisms and mechanical transmissions. Editura Universitatii Transilvania din Brasov, (in Romanian) (2006)

14. S.S. Ghimisi, Design of mechanical transmissions, Editura Didactica si Pedagogica, Bucuresti, (in Romanian), (2005)

15. I. Ionita, Quality Management and Value Engineering, Editura ASE Bucuresti, (in Romanian), (2008)

16. S.S. Ghimisi, Elements of tribology, Editura Matrixrom, Bucuresti, (in Romanian). (2005) 